Supplement of Arch. Anim. Breed., 59, 453-459, 2016

http://www.arch-anim-breed.net/59/453/2016/

doi:10.5194/aab-59-453-2016-supplement

(C) Author(s) 2016. CC Attribution 3.0 License.

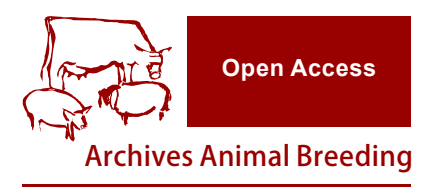

(c) (i)

Supplement of

\title{
Effect of the glycogen synthase 1 (GYS1) mutation on performance traits in 169 Noriker draft horse stallions - a retrospective study
}

Thomas Druml et al.

Correspondence to: Thomas Druml (thomas.druml@vetmeduni.ac.at)

The copyright of individual parts of the supplement might differ from the CC-BY 3.0 licence. 
Table S1 Score sheet for Noriker stallions in the obligatory standardized stationary 30-day performance test. During the four week training block the stallions get evaluated weekly in four discipline riding, driving, working and heavy load lodging. The training block comprises 19 scored traits. In the final test the stallions are being judged in 13 traits from four disciplines riding, driving, working and heavy load lodging (testing block). The final performance score is the weighted sum of all single traits from both blocks.

\begin{tabular}{|c|c|c|c|}
\hline & Disciplines & Traits & Weighting in $\%$ \\
\hline \multicolumn{4}{|l|}{ Training block } \\
\hline & \multirow[t]{7}{*}{ Riding } & Kindness & 5.00 \\
\hline & & Willingness to learn & 5.00 \\
\hline & & Performing capability & 5.00 \\
\hline & & Walk & 1.50 \\
\hline & & Trot & 1.50 \\
\hline & & Galopp & 1.50 \\
\hline & & Riding ability & 1.50 \\
\hline & \multirow[t]{6}{*}{ Driving } & Kindness & 5.00 \\
\hline & & Willingness to learn & 5.00 \\
\hline & & Performing capability & 5.00 \\
\hline & & Walk & 5.00 \\
\hline & & Trot & 5.00 \\
\hline & & Driving ability & 5.00 \\
\hline & \multirow[t]{3}{*}{ Working } & Drafting manner & 2.00 \\
\hline & & Concentration & 1.25 \\
\hline & & Kindness & 1.25 \\
\hline & \multirow[t]{3}{*}{ Heavy load lodging } & Drafting manner & 2.00 \\
\hline & & Willingness to work & 1.25 \\
\hline & & Kindness & 1.25 \\
\hline \multicolumn{4}{|l|}{ Testing block } \\
\hline & \multirow[t]{4}{*}{ Riding } & Walk & 2.00 \\
\hline & & Trot & 2.00 \\
\hline & & Galopp & 2.00 \\
\hline & & Riding ability & 2.00 \\
\hline & \multirow[t]{3}{*}{ Driving } & Walk & 5.67 \\
\hline & & Trot & 5.67 \\
\hline & & Driving ability & 5.67 \\
\hline & \multirow[t]{3}{*}{ Working } & Drafting manner & 3.50 \\
\hline & & Concentration & 2.00 \\
\hline & & Kindness & 2.00 \\
\hline & \multirow[t]{3}{*}{ Heavy load lodging } & Drafting manner & 3.50 \\
\hline & & Willingness to work & 2.00 \\
\hline & & Kindness & 2.00 \\
\hline Final performan & core & Weighted total score & 100 \\
\hline
\end{tabular}

